\title{
Observations of Rotation in Binary Stars
}

\author{
Gloria Koenigsberger \\ Centro de Ciencias Físicas UNAM, Apdo. Postal 48-3, Cuernavaca, \\ Mor. 62251
}

\begin{abstract}
A brief overview of the available information of $v \sin i$ and stellar radii in binares is presented.
\end{abstract}

\section{Rotation in Binary Stars}

Binary systems are in equilibrium when the rotational period $\left(\mathrm{P}_{\text {rot }}\right)$ of both stars coincides with the orbital period $\left(\mathrm{P}_{\text {orb }}\right)$, the orbit is circular $(\mathrm{e}=0)$, and the rotation axes are perpendicular to the orbital plane. When these conditions are not satisfied, stellar oscillations are excited. A general observational conclusion is that short-period binaries are synchronized $\left(\mathrm{P}_{\text {rot }}=\mathrm{P}_{\text {orb }}\right)$. For example, B-type primary stars in binaries with $\mathrm{P}<2.5$ days are synchronized, while those with $2.5<\mathrm{P}<5$ are nearly synchronized, within a factor of 2 (Abt et al. 1990). In order to determine whether a binary system is synchronized, the equatorial rotational velocity, $\mathrm{V}_{\mathrm{e}}$ and the radius of the star, $\mathrm{R}_{*}$ are required, in addition to $\mathrm{P}_{\text {orb }}$. The most difficult of these parameters to determine is $\mathrm{V}_{\mathrm{e}}$, since the observations only provide $v \sin i$, which requires a knowledge of the orbital inclination angle. Furthermore, the spectral line-widths which are used to determine $v \sin i$, may have contributions from other broadening sources, such as turbulence or wind velocity gradients (Howarth et al. 1997; Massa et al. 1992; Kudritzki 1992) and thus do not provide a straightforward measure of the true projected equatorial velocity $v_{\mathrm{e}} \sin i$. In this paper we present a brief overview of $v \sin i$ in binares, and focus on the problems associated with the determination of whether a given binary system is synchronized.

The largest source of $v \sin i$ in general is the recent catalogue of Glebocki et al. (2000), which contains a compilation of 17490 values of the projected equatorial velocity for about 12000 stars of all spectral types and luminosity classes. It includes the data from the catalogue of Uesugi and Fukuda (1982), which provides mean $v \sin i$ data on the old Slettebak system for 6472 stars. The Glebocki et al. compilation lists the method by which the value of $v \sin i$ was determined and the corresponding references. A comparison between the Glebocki et al. compilation and the Batten et al. (1989) binary star catalogue indicates that the former contains at least 720 binaries, for which 1731 values of $v \sin i$ are given. Many systems have measurements reported by more than one author and for a small fraction of the binaries both stars in the system have been measured.

In Figure 1 we plot the values of $v \sin i$ as a function of spectral type for the binary stars, separated according to luminosity class. It is important to 

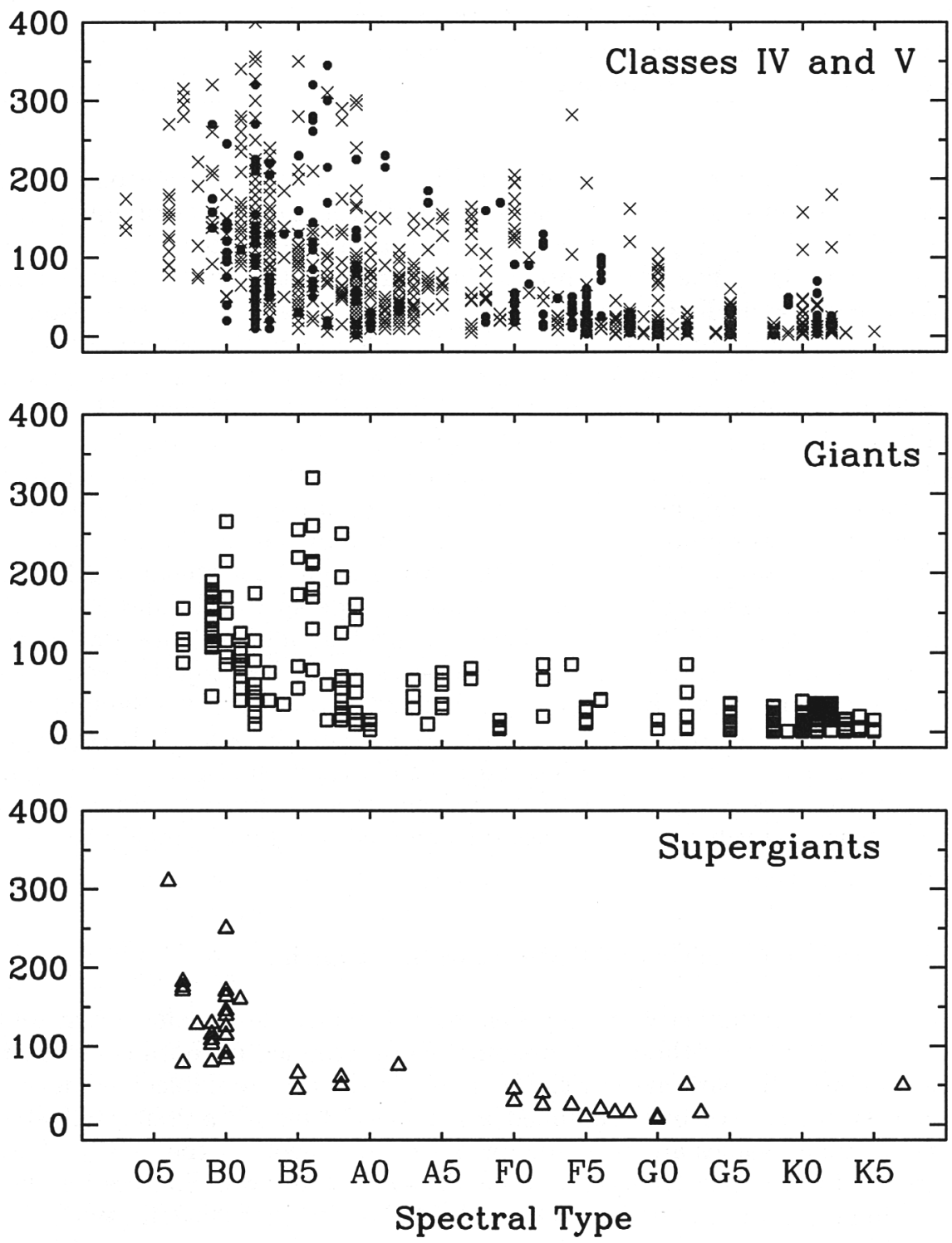

Figure 1. Observed values of $v \sin i$ as a function of spectral type, for main sequence (crosses), subgiants (filled circles), giant and supergiant stars in binary systems. 


\begin{tabular}{lllrr}
\hline Year & Author & $\begin{array}{l}\text { Description of stars in } \\
\text { the list giving } v \sin i\end{array}$ & $\begin{array}{r}\text { Number } \\
\text { of stars }\end{array}$ & $\begin{array}{r}\text { Number } \\
\text { in binaries }\end{array}$ \\
\hline 1994 & Pan et al. & m $_{v}<9.9 ; 0.5<\mathrm{P}<100 \mathrm{~d}$ & 99 & 99 \\
1995 & Abt \& Morrell & A-type stars & 1700 & $\sim 500$ \\
1997 & Howarth & O-type stars with IUE & 373 & 28 \\
2000 & Glebocki et al. & compilation O through K-types & 12000 & $>720$ \\
2002 & Gies & compilation OB binaries & - & 39 \\
2002 & Abt et al. & nortern B-stars & 1092 & 219 \\
\hline
\end{tabular}

note that although the data presented in Fig. 1 are very heterogeneous, due to the many different observational conditions and measurement techiniques, they are useful for gaining a general overview of rotation characteristics in a large sample of binaries. The plot contains data for 38 O-type stars, 248 B-stars, 183 A-type stars, 94 F-stars, 80 G-stars, and $77 \mathrm{~K}$-stars. The stars listed in Batten et al. (1989) are mostly stars with sharp lines and this selection effect is evident in Fig. 1, where few binary stars with $v \sin i>300 \mathrm{~km} \mathrm{~s}^{-1}$ are present. In addition, the absence of O-type stars with small values of $v \sin i$ (Ebbets \& Conti 1977; Howarth et al.1997) is clearly seen in Fig. 1 among the binary population. This effect is due to the additional broadening mechanisms present in the hot stars, possibly associated with turbulence or expansion of photospheric layers, a hypothesis supported by the fact that the only narrow-lined O-type star that has thus far been detected does not have a significant stellar wind. This star, MPG113 (Massey et al. 1989), has an O6-type spectrum, and lies in the Small Magellanic Cloud, where the heavy-element abundances are low. Lower metal abundances correlate with lower mass-loss rates. Its projected equatorial velocity is $35 \mathrm{~km} \mathrm{~s}^{-1}$ (see Koenigsberger, Kurucz \& Ramírez, this Symposium).

No trend of $v \sin i$ with orbital eccentricity is visible in general. However, for orbital eccentricities between $\sim 0.1$ and 0.2 the distribution of $v \sin i$ in the giant stars appears to be bi-modal, with an absence of stars having $v \sin i \sim 100 \pm 50$ $\mathrm{km} \mathrm{s}^{-1}$. Very few values of $v \sin i$ are documented for stars in highly eccentric (e>0.7) systems.

\section{Rotation Properties of Both Stars in Binaries}

Despite the large number of binary systems for which $v \sin i$ values exist, only a very small number of these have parameters determined for both components in the system. In particular, reliable stellar radii are available only for the eclipsing systems. We have compiled a list of $\sim 50$ binary systems for which $\mathrm{R}_{*}$ and/or $v \sin i$ values are available for both components of the binary. These systems contain mostly OB-type stars and have $\mathrm{P}<15$ days. Sources for these data include Levato (1976), Giuricin et al. (1984), Hilditch \& Bell (1987), Pan et al. (1994), Howarth et al. (1997), Gies (2002), and Massey et al. (2002).

In the top two panels of Figure 2 we plot the values of $v \sin i$ and $\mathrm{R}_{*}$, respectively, for both stars in each system. We find that, in general, the primary and secondary 

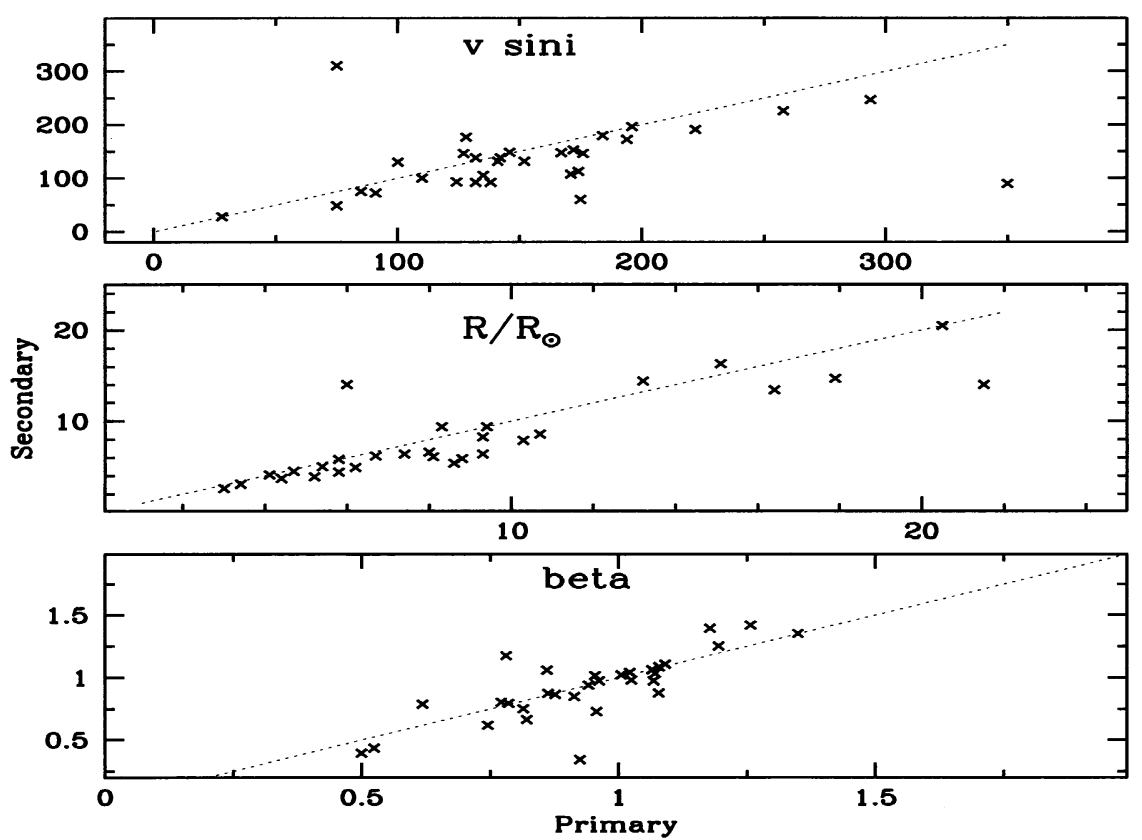

Figure 2. Observed values of $v \sin i$, stellar radius and $\beta$ for systems in which measurements for both components are available. The secondary's parameters are plotted on the ordinate. The dotted line corresponds to parameters of both components of the system being equal.

stars have similar $\mathrm{R}_{*}$ and $v \sin i$ values, although a selection effect might be present due to the difficulty in determining the parameters of stars in systems with very dissimilar stellar components. A possible trend for the secondaries to be rotating slightly slower than the primaries may be present, however. In the third panel of Figure 2 we plot the values of $\beta \sin i$. $\beta$ is defined as the ratio of the rotational angular velocity to the orbital angular velocity at periastron, and $\beta=1$ implies synchronous rotation. There is a trend for similar $\beta$ values for both members of the binary system; but a large number of the systems have $\beta$ $\sin i \neq 1$. Howarth et al. (1997) note that for 14 out of 18 individual stars in the binaries they analyzed rotate slower than the synchronous rotation (i.e., $\beta<1$. Thus, although many stars in binaries are within a factor of 2 of synchronous rotation, they are not in the equilibrium state $(\beta=1)$.

\section{X-Ray Emission and Rotation in B-Stars}

In Figure 3 we plot the ratio of ROSAT X-ray luminosity to bolometric luminosity $\left(\mathrm{L}_{\mathrm{x}} / \mathrm{L}_{\mathrm{bol}}\right.$; Berghoefer et al. 1997) as a function of $v \sin i$ for B-star binaries listed by Abt et al. (2002). Despite the large number of binaries ( 219) included in this catalogue, only a small number of these were detected by ROSAT. Binaries with eccentric orbits $(e>0.10)$ are plotted with different symbols from binaries with circular or nearly circular orbits. Two features are worth noting in 


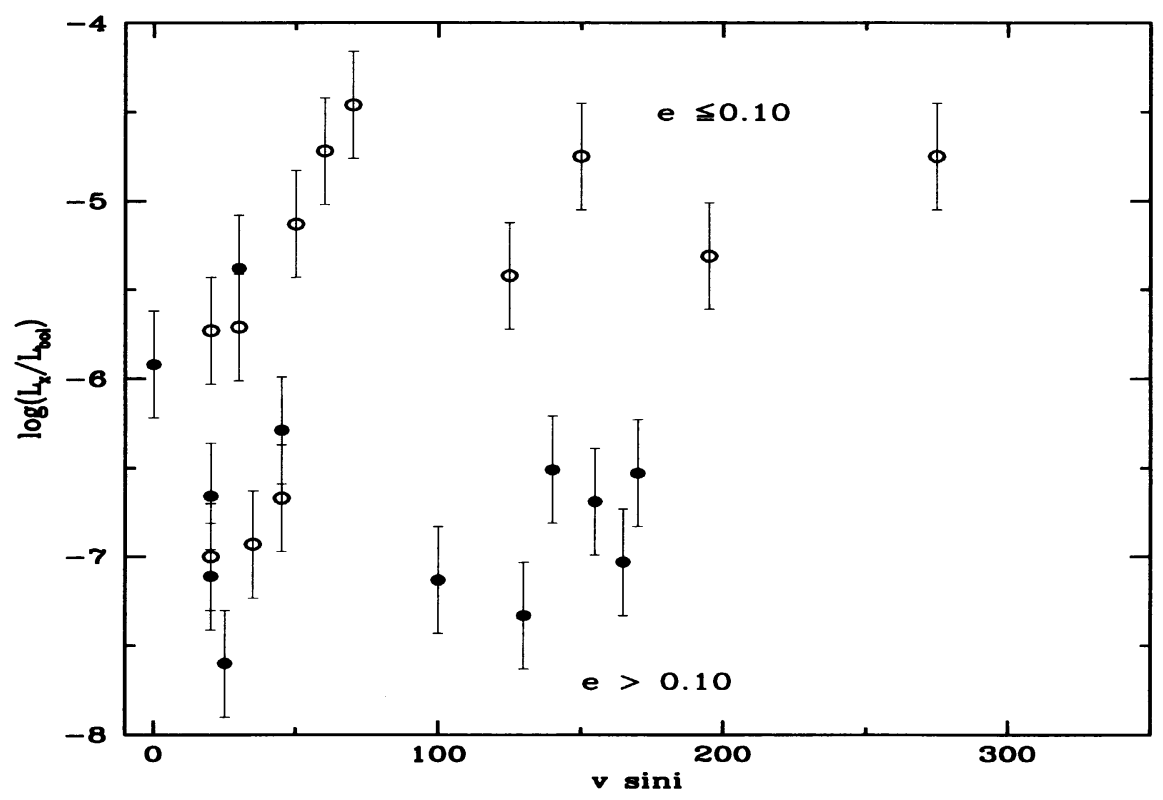

Figure 3. Observed values of X-ray luminosity as a function of $v \sin i$ for a sample of B-type stars. Note that binaries with nearly circular orbits (open symbols) have, on the average, significantly larger values of $\mathrm{L}_{\mathrm{x}} / \mathrm{L}_{\mathrm{bol}}$ than binaries with eccentric orbits (filled-in symbols). The two exceptions of eccentric systems with high X-ray luminosities are chemically peculiar stars.

Fig. 3: 1) there is a clear division between the circular and the eccentric binaries, with the $\mathrm{e}<0.10$ systems having larger $\mathrm{L}_{x} / \mathrm{L}_{b o l}$ in general; and 2) there is a trend for increasing $\mathrm{L}_{\mathrm{x}} / \mathrm{L}_{\mathrm{bol}}$ with increasing projected equatorial velocity in the circular orbit systems. The first of these features may be due to the fact that binaries with circular orbits have short periods, in general, and thus have small orbital separations. In a separate paper (Koenigsberger, Haro \& Juárez, this Symposium), we argue that stars in non-synchronous binaries may have larger mass-loss rates than stars in equilibrium configurations, and thus, have stronger wind-wind collisions which, in turn, would be responsible for the observed X-ray luminosity.

\section{Stellar Radii in Binary Systems}

We have compiled a list of 140 radius determinations for 63 different binary systems (several stars have multiple radius determinations) which are plotted in Figure 4. The large crosses are theoretical radii for single stars (Schmitd-Kaler 1982). The dash line in Figure 4 is a least mean squares fit to the main sequence data between $\mathrm{O} 6$ and $\mathrm{B} 5$, and it lies below the theoretical values for single stars. This plot also shows a large spread in radii for main sequence binaries hotter than about B1. For example, at $\mathrm{O} 6$ the range in observationally-determined 


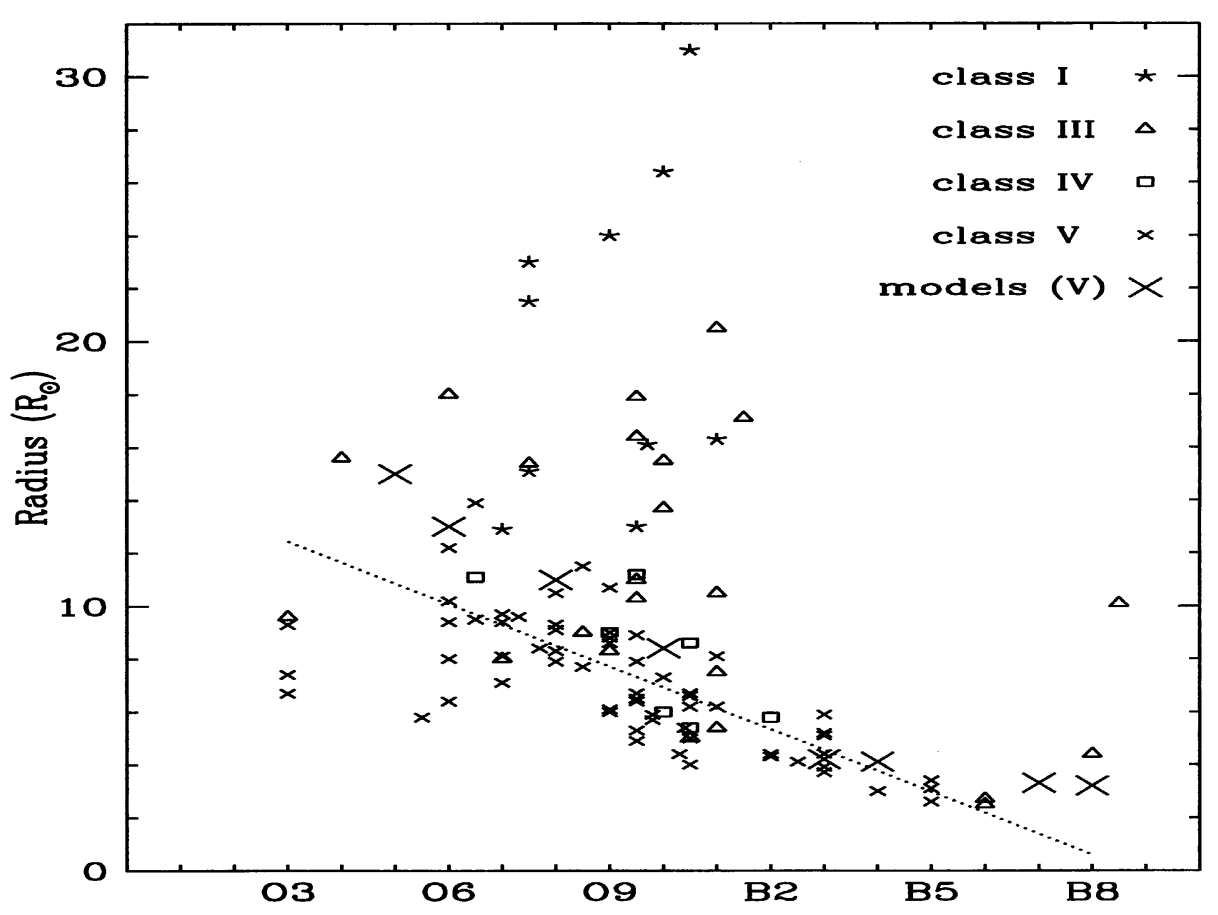

Figure 4. Values of compiled stellar radii in binary system stars (small symbols), as a function of spectral type, and theoretical values (large symbols). Different luminosity classes are plotted with different symbols. The dash line is a least-mean-squares fit to the Main Sequence data between O6-B8.

radii is 6-12 $R_{\odot}$. This spread in radii is reminiscent of the spread in absolute visual magnitudes that is observed in the Galaxy and the LMC for O3V stars (Crowther \& Dessart 1998). It is interesting to note, however, that most of the low-lying O3-O6 stars belong to the R136 cluster in the Large Magellanic Cloud, while the rest of the stars plotted in this figure are Galactic objects. The R136 binaries are eclipsing (Massey et al. 2002), and hence, the radius determination appears to be quite reliable. On the other hand, the average $\mathrm{M}_{V}$ of the LMC O3V-stars is somewhat fainter than their Galactic counterparts, so the radii of the R136 stars might be systematically smaller than the Galactic stars. However, given the small number of stars plotted in Figure 4, it is premature to draw any conclusions at this time.

\section{Conclusions}

A large number of binary systems are known for which a measurement of the $v \sin i$ parameter is available. However, only relatively small numbers of these values constitute homogeneous sets. Of these systems, an even fewer number have measurements of both stars in the system. There is a systematic trend 
for stellar radii of hot stars in binaries to be smaller than expected from an extrapolation of cooler star radii and from theoretical values for single stars. An understanding of this issue is of interest, particularly since the stellar radius is a crucial ingredient in determining whether a given binary system has achieved an equilibrium configuration.

Acknowledgements I would like to express my gratitude to Doug Gies and Laura Penny for helpful discussions, and to Kaike Pan for providing a copy of his $v \sin i$ values. This work was supported by CONACYT grant 36569 .

\section{References}

Abt, H.A., Gomez, A.E., \& Levy, S.G. 1990, ApJS 74, 551

Abt, H.A., Levato, H,. \& Grosso, M. 2002, ApJ 573, 359

Abt, H.A., \& Morrell, N. 1995 ApJS 99, 135

Batten, A.H., Fletcher, J.M., \& MacCarthy, D.G. 1989, Eighth Catalogue of the Orbital Elements of Spectroscopic Binary Systems (Publ. Dom Astrophys. Obs. Victoria 17 ; Victoria:DOA)

Berghoefer, T.W., Schmitt, J.H.M.M., Cassinelli, J.P. 1996, A\&AS 118, 481

Carroll,B.W., \& Ostlie, D.A. 1996, Modern Astrophysics, Addison-Wesley Pub. Company, Inc., pp. A-13

Crowther, P.M. \& Dessart, L. 1998, MNRAS 296, 622.

Gies, D. 2002, in IAU Symposium \# 212, van der Hucht, Herrero \& Esteban (ed.), in press

Giuricin et al. 1984a, A\&A 131,152

Giuricin et al. 1984b, A\&A 135, 393

Glebocki R., Gnacinski P., Stawikowski A., 2000, Acta Astron. 50, 509

Hilditch, Bell, 1987, MNRAS 229, 529

Kudritzki, R.P. 1992, A\&A 266, 395

Levato, H. 1976, ApJ 203, 680

Massa D., Shore, S.S., Wynne D. 1992, A\&A 264, 169

Massey, P. M., Parker, J.W., \& Garmany, C. 1989, AJ 98, 1305

Massey, P.M., Penny, L.R., \& Vukovich, J. 2002 ApJ 565, 982

Pan K., Tan, H. \& Wang, X. 1994, Chin. A\&A 18, 415

Schmitdt-Kaler, Th., 1982, "Physical Parameters of the Stars", Springer-Verlag, Berlin Slettebak, A. and Howard, R. F. 1955, ApJ 121, 102

Uesugi, A. and Fukuda, I. 1982, Revised Catalog of Rotational Velocities Department of Astronomy, Kyoto Univ., Japan 